\title{
Study of autopsy diagnosis of early myocardial infarction (MI) by triphenyl tetrazolium chloride (TTC) and histopathology
}

\author{
Manish B. Shrigiriwar ${ }^{1}$, Trishul O. Padole ${ }^{2 *}$, Laxman P. Durgawad ${ }^{3}$ \\ ${ }^{1}$ Professor, ${ }^{2}$ Associate Professor, ${ }^{3}$ Medical officer, ${ }^{1-2}$ Dept. of Forensic Medicine, ${ }^{1-2}$ Shri V N Government Medical College, Yavatmal, \\ ${ }^{3}$ Civil Hospital, Sindhudurg, Maharashtra, India.
}

\section{*Corresponding Author: Trishul O. Padole}

Email: trishulopadole@gmail.com

\begin{abstract}
Introduction: Sudden death was defined as one which has taken place within 24 hours from the onset of symptoms \& signs of disease. The diagnosis of sudden death due to acute Myocardial Infarction (MI) is difficult not only for forensic expert but for pathologist as on gross examination one may not find infarct area. To determine the usefulness, efficiency and reliability of new technique (TTC), this study has been undertaken and comparative analysis of are carried out to determine MI during the autopsy along with routine histological examination by H\&E stain.

Material and Methods: Total 107 hearts were divided into 2 groups - 1) Control group - known cases of MI and ischemic heart disease. 2) Negative control group - cases of sudden natural death, as phyxial deaths and accidental traumatic cases. The heart slices are incubated in $1 \%$ soln. of TTC in phosphate buffer at $\mathrm{pH} 8.5$ at approximately $37^{\circ} \mathrm{c}$ for $20-30 \mathrm{~min}$. Unstained slice is read as positive and bright pink stained is negative

Results: It is observed that in Group- 1 out of 15 cases TTC staining technique was positive in $80 \%$ cases while H\&E method was positive in $33.33 \%$ cases. Similarly in Group-2, out of 92 cases, TTC staining technique which is positive in $43.47 \%$ cases suggesting that the technique is more superior and sensitive technique to determine the cause of death as MI over H\&E method which is positive in $22.82 \%$ cases.
\end{abstract}

Conclusion: TTC staining technique is more sensitive \& efficient method than the H\&E stain.

Keywords: Heart slice, H\&E-staining, MI, Sudden death, TTC.

\section{Introduction}

Sudden unexpected death of apparently healthy persons always had a devastating impact on their families, the close persons and the society. It also pose serious problem to the treating physician if the person was admitted in the hospital for some other disease. Many a times it's difficult to find out exact cause of death in such situations. The definition of sudden death is variable and it changes according to authority and convention. It is based on the amount of time between the onset of symptoms and death. Depending on one's definition, the maximum time interval varies anywhere from 1 to 24 hours. Sudden death was defined as one which has taken place within 24 hours from the onset of symptoms and signs of disease, based on world health organization (WHO) definition. ${ }^{1}$ The term 'sudden death' generally denotes unexpected, natural non-traumatic death. WHO report states that cardiovascular disease was the leading cause of non-communicable disease deaths. There were17.5 million deaths due to cardiovascular diseases in 2012. In India there were 3.06million deaths due to cardiovascular diseases in 2012. According to WHO prediction, by $2020 \mathrm{AD}$ up to three-quarters of death in developing countries would result from non-communicable diseases and that coronary heart disease will top the list of killers. ${ }^{2}$ Coronary heart disease otherwise called as Myocardial Infarction (MI) is a dreaded complication of coronary atherosclerosis.

The diagnosis of sudden death due to acute myocardial infarction is difficult not only for forensic expert but for pathologist especially when the death has occurred within 6 hrs after myocardial infarction. On gross examination one may not find infarct area. Coronary artery disease is sometimes called 'The Captain of the Men of death' and is by far the most frequent cause of sudden and unexpected deaths which constitute a significant portion of the autopsies that are conducted by Forensic Pathologists in our country. ${ }^{3}$ The following features are characteristic of infarction in sections stained by haematoxylin and eosin. Eosinophilia, swelling of muscle-fibers, granularity of cytoplasm, blurring of cell membranes, corrugation of dead muscle fibers, increase in interstitial cells. With the introduction of gross histochemical staining technique such as TTC (Triphenyl Tetrazolium Chloride) method (This technique relies on the ability of dehydrogenase enzymes and cofactors in the tissue to react with tetrazolium salts to form a formazan pigment), it is now possible to detect myocardial infarction at a stage considerably earlier than was hitherto possible by conventional methods.

This study is being undertaken in this region as there are no other methods to diagnose the sudden natural deaths that occurred within 24 hours of incidence. In order to determine the usefulness, efficiency and reliability of this new technique (TTC) this study has been undertaken and comparative analysis of these methods are carried out to determine myocardial infarction during the medico legal autopsy along with routine histological examination by haematoxylin \& eosin stain. 


\section{Materials and Methods}

In this study (total duration 24 months) total 107 hearts were removed by Virchow's method from the dead bodies of routine medico legal autopsies which were carried out in the department of Forensic Medicine, shri vasantrao naik govt. medical college, Yavatmal. The pericardial sac is excised first. Then the heart is held at apex with left hand and lifted upwards with the knife held in horizontal position in right hand. Then the inferior vena cava, left pulmonary vein, left pulmonary artery, right pulmonary vein, ascending aorta and superior vena cava are cut through in this order. It is cut as far away as possible from the base of the heart. (Standard text - Autopsy diagnosis and technique by Otto Saphir, Third edition 1951). ${ }^{4}$

The clinical data for each case was derived primarily from evidence of prior cardiovascular and other diseases obtained from relative of deceased or friends or from police and accordingly the cases were divided into two groups:-

\section{Control group}

known cases of myocardial infarction and ischemic heart disease were included in this group which shows clinical signs of myocardial infarction along with relevant history obtained from relative of deceased and which showed coagulation necrosis of myocardium at autopsy has been labelled as control group.

Negative control group - cases of sudden natural death, asphyxial deaths and accidental traumatic cases are included in this group presuming that these might produce myocardial infarction at the terminal stage for comparative analysis.

The slice of heart is processed for TTC (Triphenyl Tetrazolium Chloride) solution. TTC (Triphenyl Tetrazolium Chloride) Solution is - 1\% TTC (Triphenyl Tetrazolium Chloride) solution was prepared by adding $1 \mathrm{gm}$ of TTC powder to $100 \mathrm{ml}$ phosphate buffer $\mathrm{pH} 8.5$ and TTC incubating reagent is prepared as -1 percent solution of Triphenyl Tetrazolium Chloride (TTC) is dissolved in phosphate butter of $\mathrm{pH}-8.5$. The $\mathrm{pH}$ alters on addition of TTC which is readjusted by addition of drops of N/10 $\mathrm{NaOH}$ solution for which $\mathrm{pH}$ paper used.

The slice of the heart is rinsed briefly in saline first \& then it is incubated in $1 \%$ soln. of TTC in phosphate buffer at $\mathrm{pH} 8.5$ at approximately $37^{\circ} \mathrm{c}$ for $20-30 \mathrm{~min}$. The slice is then incubated in 1 percent TTC solution \& is kept in the incubator for 20-30min. area of colour changes observed. If slice remain unstained it is read as positive and photograph is taken and if slice stained bright pink it is read as negative and accordingly observation is made. Then irrespective of positive $\&$ negative results at least sections were taken from wall of both ventricles \& stained with $\mathrm{H} \& \mathrm{E}$ stains method.

\section{Results}

The present study has been performed on 107 cases which were brought to shri. vasantrao naik govt. medical college and hospital, Yavatmal for routine medico legal examinations. The routine medico legal autopsies are usually performed within 24-48 hours after death, 3 cases are examined after 3 days but given normal mortuary refrigeration. Out of the total 107 cases examined, the age and sex distribution of cases studied is as given in Table 1.

Table 2, it is seen that in control group of myocardial infarction and atherosclerotic heart disease, the TTC staining technique is positive in 12 cases ( 80 percent) out of 15 cases and negative in 3 cases because TTC staining technique is positive in infarction of 5-6 hours age of infarction. While in negative control group it is positive in maximum cases in sudden natural death group in 29 cases (60.4 percent) out of 48 cases giving positive reaction suggestive of myocardial infarction and in total 40 cases (43.47 percent) out of 92 cases it gives positive results. Then irrespective of positive and negative result shown by TTC staining solution technique, the tissues are taken from 5 different sites for paraffin block preparations.

From observations of Table 3 , on histopathological examination of the tissue by haematoxyline and eosin stain in 07 (46 percent) cases in group-1 out of 15 cases the slides show coagulated necrosis, oedema and haemorrhage with waviness and thinning of fibers seen as separation of fibers suggestive of infarct is 6-12 hours. While in 6 cases (40\%) it shows necrotic fibers, these fibers exhibited increase eosinophilia, loss of striations and granularity of cytoplasm with nuclear pyknosis or karyolysis suggestive of that the age of infarct is 24hours or less and in 04 of cases necrotic fibers were clearly evident with total coagulative necrosis with increase loss of recognizable organelles suggestive of that the age of infarction is 48 hours or old. While in 6 of the cases there was no tissue changes seen suggestive of myocardial infarction. While in group-2, 27 cases (29\%) the slides show coagulated necrosis, oedema and haemorrhage with waviness and thinning of fibers seen as separation of fibers suggestive of infarct is 6-12 hours while necrotic fibers were clearly evident in 17 cases $(18 \%)$.

It is observed that in Table 4, Group-1 out of 15 cases TTC staining technique was positive in 12 cases $(80 \%)$ while haematoxyline and eosin method was positive in 05 cases $(33.33 \%)$. Hence TTC method is more sensitive to determine the diagnosis of myocardial infarction as it is positive in all the cases as compared to haematoxyline and eosin which is positive in only 5 cases. Similarly in Group2, 92 cases which has been examined by two methods TTC staining technique which is positive in 40 cases (43.47 percent) suggesting that the technique is more superior and sensitive technique to determine the cause of death as myocardial infarction over haematoxyline and eosin method which is positive in 21 cases (22.82 percent). TTC positive cases in sudden natural deaths were 29 (60.4\%) and H \& E positive cases are $14(29 \%)$ out of 48 cases. While in miscellaneous cases TTC Positive cases are $9(34 \%)$ and $\mathrm{H}$ $\&$ E positive cases are $6(23 \%)$ and in accidental deaths TTC positive cases are $2(20 \%)$ and $\mathrm{H} \& \mathrm{E}$ positive cases are 1 $(10 \%)$. Thus form above table it is observed that TTC examination revealed positive result in both control and negative control group in maximum number of cases as compared to haematoxyline and eosin method. 


\section{Discussion}

In the present study the total number 107 cases (Table 1) are examined in group-1 \& group-2 for determination of myocardial infarction. Out of this 53 cases shows the evidence of myocardial infarction indicating that incidence of myocardial infarction is prevalent in age group of 25-70 years. However in present series of cases maximum number of incidence of myocardial infarction, 17 cases (32 percent) are noticed in the age group of 51-60 years and next in the age group of $41-50$ years, 11 cases (20.75 percent), in the age group of 61-70 years 9 cases (16 percent) while least number of incidence 6 cases ( 11 percent) are seen in the age group of 20-30 years. This incidence of myocardial infarction in the age group is observed in the present series are not tally with the observations made by the some author such as $\mathrm{G}$ Baroldi et $\mathrm{al}^{6}$ observed that maximum number of cases (41 cases) in his study falls between 60-69 years and 18 cases in age group of 50-59 years while Rissanen M Romo et $\mathrm{al}^{7}$ had also noticed that the maximum number of cases (56 cases) are of myocardial infarction in the age group of 55-64 years and 30 cases in the age group of 45-54 years. This difference might be due to the fact that in our country the eating habits, indiscriminate use of tobacco and lack of physical activity in the people increases the chances of getting this malady in the earliest age group. Banshidhar Gupta $^{8}$ in 2013-This study tally with study conducted by author where maximum 25 cases were found in the age group 51-60 years and least cases 3 cases in the age group 20-30 years.

In the present study, out of 15 cases (Table 2) with clinical history of myocardial infarction, in 12 cases the heart slice were positive on gross examination by TTC (Triphenyl Tetrazolium Chloride) staining technique and 3 cases were negative. The negative cases in this group are not positive with the TTC staining even though it is a known case of myocardial infarction. This is due to the fact that TTC does not give positive results in cases where the age of infarct is of 5-6 hours duration. Another group of 92 cases with no gross pathology of heart shows positive results in 40 cases and negative result in 52 cases. Marvin et $\mathrm{al}^{9}$ in 1963 studied the method of TTC for demonstration of macroscopic identification of myocardial infarction on Guinea pigs. He examined the heart of animals at interval of 1-120 hours after coronary occlusion and necropsy done by 2-7 hours after death and his experience with 112 human hearts shown that it was possible to recognize infarct at necropsy by Triphenyl Tetrazolium Chloride method who died as early as 4-24 hours. In the present worker study the earliest time of detection of myocardial infarction by TTC method was 6 hours however by the Marvin et at the time of detection of myocardial infarction varied from 4-24 hours. Bernard Knight ${ }^{10}$ in 1967 studied the method of Triphenyl Tetrazolium Chloride to detect the early myocardial infarct and he showed that the earliest infarction can be diagnosed by 1-2 hours of time.Gradwohl ${ }^{11}$ in the year 1968 stated that normal heart muscle with active dehydrogenases acts on Triphenyl Tetrazolium Chloride to produce a bright red formazan compound. He observed that with this method an infarcted myocardium can be picked up in 4 hours as there is no deposition of the indicator because of reduced or absent enzymes in the infarcted area and area remains pale in contrast to normal stained tissue. Michael $\mathrm{C}$ Fishbein et $\mathrm{al}^{12}$ in 1981 studied on 16 dogs heart. Out of which 12 dogs killed after 6 hours and 4 dogs killed after 3 hours of occlusion. He observed that all infarcts were sharply delineated by the TTC technique. It was found that myocardial infarction show loss of staining when stained with TTC as early as 1-2 hours after the coronary occlusion. Gradwohl $^{11}$ in 1968 found on routine $\mathrm{H}$ and $\mathrm{E}$ methods the following features of myocardial infarction (Table 3).

\section{Eosinophilia}

It is most important sign of early infarction though it appears until at least 6hours, the eosinophilia may be seen in large muscles and usually goes parallel with swelling.

\section{Swelling of muscles fibers}

The normal intercellular spaces become reduced due to edema of the cells. This often occurs in large masses creating a block of dense tissue.

\section{Granularity of cytoplasm}

This is a cloudy swelling of classic cellular pathology and may be a reversible phenomenon.

\section{Blurring of cell membranes}

It occurs at the early stages long before the cell membrane begins to disintegrate due to irreversible necrosis.

\section{Corrugation}

Dead muscle fibers may be corrugated and where the bending is severe they may become pinched i.e. sharply angulated. Corrugation may appear as a normal phenomenon but in infarcted myocardium the corrugations are wide and regular and often parallel fibers are affected. A C Ritchie ${ }^{13}$ in 1990 observed that microscopically little changes can be seen in first6 hours after infarction. After 6 hours congestion and oedema begins to occur at the margins of infarct. A few hours more neutrophils begin to appear around the margin of infarct as typical acute inflammation develops around the dead muscle. In this region neutrophilic exudates is intense for the first week or so after infarction. In the present study the present worker could get oedema but could not confirm the neutrophilic exudates at the necrotic area. In comparative analysis of the 2 methods Triphenyl tetrazolium chloride staining technique and haematoxylin and eosin stain in the present it is observed that of total 52 cases show macroscopic demonstration of myocardial infarction by TTC staining technique. Out of which in 20 of the cases on routine histological examination shown evidence of necrosis on light microscopy. Banshidhar Gupta et $\mathrm{al}^{8}$ in 2013 studied on 100 hearts and histochemical study was carried out with using haematoxylin \& eosin as a routine stain and Triphenyl Tetrazolium Chloride as gross marker, $68 \%$ of cases showed positive result with TTC staining while only $25 \%$ of cases 
showed positive result with $\mathrm{H} \& \mathrm{E}$ stain. Thus in the present study (Table 4) it is observed that TTC staining technique is more reliable as it gives positive result in $60.4 \%$ as compared to haematoxyline and eosin stain which give positive results in $29 \%$ cases.

Table 1: Age and sex distribution

\begin{tabular}{|c|c|c|c|}
\hline Age & Male & Female & Total \\
\hline $20-30$ & 12 & 08 & 20 \\
\hline $31-40$ & 24 & 01 & 25 \\
\hline $41-50$ & 23 & 02 & 25 \\
\hline $51-60$ & 23 & 01 & 24 \\
\hline $61-70$ & 10 & 02 & 12 \\
\hline $71-80$ & 01 & 00 & 01 \\
\hline Total & 93 & 14 & 107 \\
\hline
\end{tabular}

Table 2: Observation by TTC staining technique

\begin{tabular}{|l|l|c|c|c|}
\hline \multirow{4}{*}{ Group-1 } & \multicolumn{1}{|c|}{ Control Group } & No. of cases & Positive & Negative \\
\cline { 2 - 5 } & Myocardial Infarction & 11 & 09 & 02 \\
\cline { 2 - 5 } & Atherosclerotic Heart Disease & 04 & 03 & 01 \\
\cline { 2 - 5 } & Total & 15 & $12(80 \%)$ & 03 \\
\hline \multirow{5}{*}{ Group-2 } & Sudden Natural Death & 48 & $29(60.4 \%)$ & 19 \\
\cline { 2 - 5 } & Hanging & 06 & 00 & 06 \\
\cline { 2 - 5 } & Drowning & 02 & 00 & 02 \\
\cline { 2 - 5 } & Accidental deaths & 10 & $02(20 \%)$ & 08 \\
\cline { 2 - 5 } & Miscellaneous & 26 & $09(34 \%)$ & 17 \\
\cline { 2 - 5 } & Total & 92 & $40(43.74 \%)$ & 52 \\
\hline
\end{tabular}

Table 3: Observation by $\mathrm{H}$ and $\mathrm{E}$ staining method

\begin{tabular}{|c|c|c|c|c|c|c|}
\hline \multirow[t]{4}{*}{ Group-1 } & Cause of death & $\begin{array}{c}\text { Number } \\
\text { of } \\
\text { cases }\end{array}$ & $\begin{array}{c}\text { Waviness \& } \\
\text { thinning of } \\
\text { fibers, } \\
\text { coagulation, } \\
\text { necrosisintercel } \\
\text { lular oedema }\end{array}$ & $\begin{array}{l}\text { Necrotic } \\
\text { changes }\end{array}$ & $\begin{array}{c}\text { Total coagulative } \\
\text { necrosis with loss } \\
\text { of nuclei \& } \\
\text { striations }\end{array}$ & $\begin{array}{l}\text { Tissue changes not } \\
\text { suggestive of } \\
\text { myocardial } \\
\text { infarction }\end{array}$ \\
\hline & Myocardial infarction & 11 & 05 & 04 & 03 & 06 \\
\hline & $\begin{array}{l}\text { Atherosclerotic } \\
\text { Heart Disease }\end{array}$ & 04 & 02 & 02 & 01 & 00 \\
\hline & Total & 15 & 07 & 06 & 04 & 06 \\
\hline \multirow[t]{6}{*}{ Group-2 } & $\begin{array}{l}\text { Sudden Natural } \\
\text { Death }\end{array}$ & 48 & 19 & 12 & 06 & 25 \\
\hline & Hanging & 06 & 00 & 00 & 00 & 06 \\
\hline & Drowning & 02 & 02 & 02 & 00 & 02 \\
\hline & Accidental deaths & 10 & 01 & 01 & 00 & 08 \\
\hline & Miscellaneous & 26 & 05 & 02 & 02 & 17 \\
\hline & Total & 92 & 27 & 17 & 08 & 58 \\
\hline
\end{tabular}

Table No. 4: comparative analysis of TTC and H \&E methods

\begin{tabular}{|c|l|c|c|c|}
\hline Group-1 & Cause of death & No. of cases & TTC staining positive & H \& E method positive \\
\cline { 2 - 5 } & Myocardial Infarction & 11 & 09 & 04 \\
\cline { 2 - 5 } & Atherosclerotic Heart disease & 04 & 03 & 01 \\
\cline { 2 - 5 } & Total & 15 & $12(80 \%)$ & $05(33.33 \%)$ \\
\hline \multirow{5}{*}{ Group-2 } & Sudden Natural Death & 48 & $29(60.4 \%)$ & $14(29 \%)$ \\
\cline { 2 - 5 } & Hanging & 06 & 00 & 00 \\
\cline { 2 - 5 } & Drowning & 02 & 00 & 00 \\
\cline { 2 - 5 } & Accidental deaths & 10 & $02(20 \%)$ & $01(10 \%)$ \\
\cline { 2 - 5 } & Miscellaneous & 26 & $09(34 \%)$ & $06(23 \%)$ \\
\cline { 2 - 5 } & Total & 92 & $40(43.74 \%)$ & $21(22.82 \%)$ \\
\hline
\end{tabular}




\section{Conclusion}

In the present series of study, the incidence of myocardial infarction is prevalent in males as compared to females and also it is more in 40-60 years of age as compare to Western countries. From comparative analysis to determine the sensitivity and efficiency of different techniques such as TTC staining technique and Haematoxy line and Eosin stain to determine myocardial infarction at necropsy, it has been noticed that TTC staining technique is more sensitive and

Acknowledgement: None

\section{Conflict of interest: None.}

\section{Source of funding: None.}

\section{References}

1. Reddy KS. Cardiovascular disease in non-Western countries. N Engl J Med. 2004;350:2438-510.

2. Global status report on non-communicable diseases 2010 . WHO. https://www.who.int $>\mathrm{nmh}>$ publications > ncd_report_full_en.

3. Saukko Pekka, Knight Bernard. Knights Forensic Pathology.3 Ed. New York Oxford University Press; 2004 p. 492-08.

4. Otto Saphir. Autopsy Diagnosis and Technic.1961. 4th edition. New York. Hoeber and Harper.

5. Robbins, Kumar \&Kotran. Pathologic Basis of Disease (8th Edition). W. A.Saunders Publication, Philadelpia.

6. G Barroldi, F Radice G, A Leone. Morphology of Acute Myocardial Infaction in relation to Coronary Thrombosis. American Heart Journal.1974;87(1):65-5.

7. V. Rissanen, M.Romo, P.Siltanen. Prehospital sudden death from ischaemic heart disease. A post mortem study. British Heart Journal.1978;40:1025-33.

8. Banshidhar Gupta, Hetal Gohel, Nandini Desai, Shital Dodhia. Post mortem study of heart in cases of sudden cardiac death using Triphenyl tetrazolium chloride and haematoxylin \& eosin stain. Indian J Appl Basic Med.2013:25.

9. Marvin M. Nachlas, M.D.; Theodor K. Shnitka, M.D. Macroscopic Identification of Early Myocardial Infarcts by Alterations in Dehydrogenage Activity. The American $J$ Pathol. April 1963;42(4):379-05.

10. Bernard Knight. Early myocardial infarction. Practical Methods for its Demonstration. J Forensic Med.1967;14 (3):101-07.

11. Gradwohl. Gradwohl‘s Legal Medicine. 1968, llnd Edition. John Wrights \&Sons Ltd. Publications, U.S.A.

12. Michael C. Fishbein MD, Derek Maclean. Peter R. Maroko MD. The histopathologic evolution of Myocardial Infarction. Chest.1978;73(6):843-9.

13. Boyd's Text book of Pathology (9th Edition). 1990,2. Philadelphia, London. P223-27.

How to cite this article: Shrigiriwar MB, Padole TO, Durgawad LP. Study of Autopsy Diagnosis of Early Myocardial Infarction (MI) by Triphenyl Tetrazolium Chloride (TTC) and Histopathology. Indian J Forensic Community Med 2019;6(4):220-4. efficient method than the Haematoxy line and Eosin stain. The microscopic study by $\mathrm{H}$ and $\mathrm{E}$ stain is useful as a routine technique but it has the disadvantage of affecting by autolysis and its value is limited by survival time by $6-12$ hours from onset of ischemia until morphological changes can be detected. We recommend the staining technique to be included in the autopsy of sudden deaths. Therefore the excess of needless routine test could be avoided. 